\title{
Immunoreactivity of ATF-2 and Fra-2 in human dental follicle
}

\author{
Ilker Bolat, Nurullah Keklikoglu
}

Istanbul University, Faculty of Dentistry, Department of Histology and Embryology, Istanbul, Turkey

\begin{abstract}
It is asserted that epithelial rests in dental follicle (DF) existing around the impacted teeth in adults are effective in cyst formation. In this study, it is intended for determining and comparing the immunoreactivity (IR) ratio of ATF-2 and Fra-2 proteins, the members of Activator Protein-1 (AP-1) family which regulates important cellular activities such as growth, proliferation and differentiation, in DF epithelial cells (EC) and connective tissue cells (CC). In this study, ATF-2 and Fra-2 immunoreactivity (ATF-2-IR and Fra-2-IR) in EC and CC in DF tissues obtained from 30 patients were analyzed by using immunohistochemical method. Ratios of ATF-2-IR positive cells were found $17.36 \pm 9.55 \%$ in EC, $27.27 \pm 14.86 \%$ in CC and ratios of Fra-2-IR positive cells were found $20.04 \pm 11.47 \%$ in EC, $16.71 \pm 9.05 \%$ in CC. In the statistically comparison performed; significant differences were found between EC and CC in terms of both ATF-2-IR ( $p<0.001)$ and Fra-2IR $(\mathrm{p}<0.05)$. In EC, no significant difference was found between ATF-2-IR and Fra-2-IR ( $>0.05)$, whereas significant difference was found between ATF-2-IR and Fra-2-IR in CC $(\mathrm{p}<0.001)$. According to these data, it can be suggested that Fra-2 protein may be more effective than ATF-2 protein in cyst formation originated from EC of DF. Besides, finding that ATF2-IR and Fra-2-IR are different in CC although similar in EC shows that AP-1 members can be expressed at different ratios in same tissues.
\end{abstract}

Key words: dental follicle, AP-1, ATF-2, Fra-2, epithelial rests

\section{Introduction}

In the cells facing with an extracellular stimulus, the first genes which responding to the stimulus are immediate early genes (IEGs) and the proteins which are members of transcription factor activator protein-1 (AP-1) are the nuclear products of this group of genes. Function of transcription factors is to regulate the gene transcription. Many cellular function take place depending on transcription factors [1-3]. AP-1 transcription complex functions in several cellular processes such as growth, proliferation, differentiation, apoptosis and oncogenic transformation [4]. AP1 is an inducible transcription factor and consists of multiple protein complexes like many transcription factors $[3,5]$.

AP-1 transcription factor complex mainly consists of Fos protein family (c-Fos, FosB and its smaller splice variant FosB2, Fra-1 and Fra-2), Jun protein family (c-Jun, JunB and JunD), activating transcrip-

Correspondence: N. Keklikoglu, Istanbul Universitesi, Dishekimligi Fakultesi, Histoloji ve Embriyoloji BD, Capa, Istanbul, Turkey; tel.: (+90212) 4142020-30221,

e-mail: nkeklik@istanbul.edu.tr tion factor (ATF) subfamily (ATFa, ATF-1 ATF-2, and ATF-3) and Jun dimerization protein (JDP) subfamily (JDP-1 and JDP-2) members. All AP-1 proteins are characterized by a basic leucine-zipper region [6-9]. AP-1 proteins are unite through these specific points located on them and compose dimmers $[3,6]$. Also, they bind to the promoter regions on DNA containing TPA (12-O tetradecanoylphorbol 13-acetate) response element (TRE; TGAC/GTCA) by these regions to regulate the expression of last gene responses and make changes in the cell $[6,8]$.

ATF-2 and Fos proteins are commonly seen in pathological processes. Recent studies have shown that over-expression of ATF-2 increases the proliferation in human and mouse cancer cells [10,11]. Again, in studies performed, it is suggested that over-expression of Fra-2 is associated with the phenotype of aggressive tumour at some cancers and may be related to the progress of some cancer types and Fos-related antigen-2 (Fra-2) is showed to be an important AP-1 transcription factor in differentiating cells $[4,8,12]$.

During tooth eruption, c-Fos is expressed from dental follicle (DF) cells and this expression has an important role in tooth eruption [13-15]. 
Table 1. Percentage of cells with positive ATF-2 and Fra-2 immunostaining in epithelial cells (EC) and connective tissue cells (CC) in human dental follicle.

\begin{tabular}{|c|c|c|c|}
\hline \multirow{2}{*}{ Antibodies } & \multicolumn{2}{|c|}{$\begin{array}{c}\text { \% Immunoreactive cells } \\
(\text { mean } \pm \text { SD) }\end{array}$} & $\begin{array}{c}\text { Statistical } \\
\text { significance }\end{array}$ \\
\cline { 2 - 4 } & $\mathrm{EC}$ & $\mathrm{CC}$ & $\mathrm{p}^{*}$ \\
\hline ATF-2 & $17.36 \pm 9.55$ & $27.27 \pm 14.86$ & 0.0001 \\
\hline Fra-2 & $20.04 \pm 11.47$ & $16.71 \pm 9.05$ & 0.0222 \\
\hline $\mathrm{p}^{*}$ & 0.1962 & 0.0002 & \\
\hline
\end{tabular}

* paired-samples t-test.

DF is an ectomesenchymal tissue which covers the surrounding of tooth germ and includes several cell types. In human, DF involves cementoblast precursors, periodontal ligament cells and osteoblasts [16-18] and forms cementum, periodontal ligament and parts of alveolar bone that are close to tooth $[19,20]$.

Since DF present around the unerupted impacted teeth in adults can undergo some pathological changes especially dentigerous cyst (follicular cyst), it is argued that it is required to be removed surgically during extraction of impacted teeth [21].

Follicular cysts are claimed to be originating from epithelial rests in DF cells [22]. It is stated that apoptosis and cell proliferation are effective in formation of dentigerous cysts surrounding the crown of impacted teeth [20].

However, there is not sufficient number of research with respect to the expressions of AP-1 member proteins and their roles in the pathological development in cystic structures developing from the epithelial cell rests contained by DF around the teeth which have completed their development but not erupted.

In this study, it is intended for determining and comparing immunoreactivity (IR) of ATF-2 and Fra-2 proteins, the members of Activator Protein-1 (AP-1) family which plays important roles in many cellular activities such as proliferation and apoptosis that is also required for cyst development in tissues forming DF present around the impacted teeth.

\section{Materials and methods}

Tissue samples. In this study, DFs which are present around unerupted impacted teeth and which have been extracted surgically together with these teeth were used, however DFs which have been found to turn into cystic structure clinically and radiologically were not used. DFs were obtained from 30 patients (17 males, 13 females) whose ages were between 18 and 39 (average of age 25.86 \pm 6.95 ).

The freshly obtained DFs were fixed in $10 \%$ neutral buffered formalin for 12-18 hours. After fixation process, the tissues; for the purpose of removing fixation solution, were washed in PBS (Phosphate Buffered Saline) solution (pH 7.3, Zymed, CA) which was changed 5-6 times.
Washed tissues were dehydrated by taking into alcohol series at the rates increasing respectively. After being become transparent through xylene, DFs were embedded in paraffin blocks and sections with the thickness of 5-6 microns were taken. As 6 sections for each protein to be examined from every tissue block, $12 \mathrm{sec}-$ tions were taken (excluding sections to be used for negative control, total 360 sections). After sections were mounted on glass slides, for the purpose of removing from paraffin, they were taken into xylene solution twice as 30 minutes in each one, they were rehydrated by taking into alcohol series at the decreasing rates for preparing to immunohistochemical staining.

Immunohistochemistry. After the tissue sections were rehydrated, they were heated in sodium citrate buffer ( $\mathrm{pH}$ 6.0) (ZYMED, CA) at $95^{\circ} \mathrm{C}$ for 15 minutes for antigen retrieval. Then, they were left for cooling for 25 minutes.

For blocking endogenous peroxidase activity, sections were incubated for 10 minutes at room temperature in 3\% hydrogen peroxide solution. After sections were washed with PBS for 10 minutes, for the purpose of preventing non-specific background staining, they were incubated for 10 minutes at room temperature in serum blocking solution (Histostain-Plus Bulk Kit, ZYMED, CA).

Then, tissue sections were incubated for 6 hours with ATF-2 (ATF-2 (C-19): sc-187, Santa Cruz Biotechnology, Inc., CA) and Fra-2 (Fra-2 (L-15): sc-171, Santa Cruz Biotechnology, Inc., CA) antibodies which are primary antibodies (rabbit polyclonal antibodies) composed against the proteins to be determined.

Primary antibodies were diluted (ATF-2; 1:100 and Fra-2; 1:80) by antibody diluent solution (ZYMED, CA) prior to use. Preparations reserved for negative control were incubated with non-immune rabbit serum instead of primary antibodies. After primary antibodies, the sections were incubated for 10 minutes with biotinylated secondary antibody (Histostain-Plus Bulk Kit, ZYMED, CA).

Immunoreactivity was determined through the incubation of tissues with horseradish-peroxidase (HRP)-streptavidin complex (Histostain-Plus Bulk Kit, ZYMED, CA) for 10 minutes and aminoethyl carbazole substrate (AEC) chromogen (AEC (red) Substrate Kit, ZYMED, CA) for 15 minutes as a marker. Counterstain was applied to the sections for 0,5-2 minutes with Mayer's hematoxylin.

After sections were washed in distilled water which was changed 3 times, they were closed with mounting media (UltraMount Plus, Lab Vision Corporation, CA) and examined by light microscope and were photographed.

Ethical issues. The study protocol was approved by the Ethics Committee of Istanbul University Istanbul Faculty of Medicine (2006/2059).

Statistical analysis. For statistically comparing ATF-2 and Fra-2 immunoreactivity (ATF-2-IR and Fra-2-IR) in epithelial cells (EC) and connective tissue cells (CC) of DFs, paired samples t-test was used. Statistically significance degree was accepted as $\mathrm{p}<0.05$.

\section{Results}

In EC and CC forming DF, both ATF-2-IR and Fra-2IR positive cells were detected in different proportions. The percentage of EC and CC which were ATF2-IR and Fra-2-IR positive within the cell group they belong to were calculated.

ATF-2-IR positive cell rates were calculated as $17.36 \pm 9.55 \%$ in EC, and as $27.27 \pm 14.86 \%$ in CC. Fra2-IR positive cell rates were calculated as 

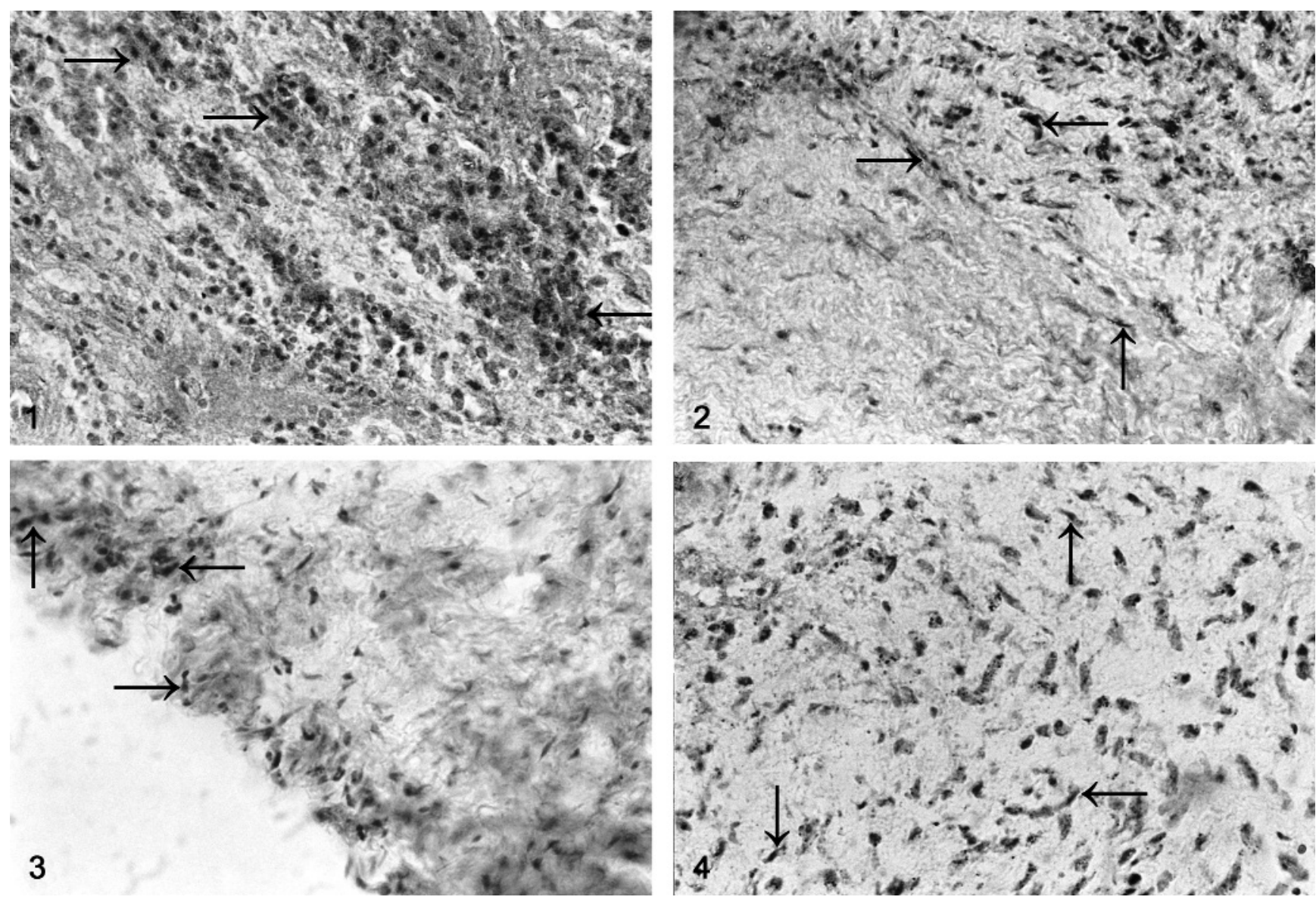

Fig. 1. ATF-2 immunoreactivity in epithelial cells of human dental follicle (arrows, original magnification $\times 400$ ). Fig. 2. ATF-2 immunoreactivity in connective tissue cells of human dental follicle (arrows, original magnification $\times 400$ ). Fig. 3. Fra- 2 immunoreactivity in epithelial cells of human dental follicle (arrows, original magnification $\times 400$ ). Fig. 4. Fra-2 immunoreactivity in connective tissue cells of human dental follicle (arrows, original magnification $\times 400$ ).

$20.04 \pm 11.47 \%$ in EC and $16.71 \pm 9.05 \%$ in CC (Table 1). In ATF-2-IR positive EC and CC, both nuclear and cytoplasmic staining were observed (Fig. 1 and 2). Nuclear staining was observed predominant in Fra-2IR positive EC, whereas both cytoplasmic and nuclear staining were observed in CC (Fig. 3 and 4).

While ATF-2-IR positive cell rate was found higher in CC than EC, Fra-2-IR positive cell rate was found higher in EC than CC (Table 1). In the statistical comparison (paired samples t-test), a highly significant difference was found between EC and CC in terms of ATF-2-IR $(p<0.001)$, whereas significant difference was found in terms of Fra-2-IR $(p<0.05)$.

In the statistical comparison of ATF-2-IR and Fra2-IR in each one of EC and CC, no significant difference was found between ATF-2-IR and Fra-2-IR in EC $(p>0.05)$, whereas a highly significant difference was found in the comparison of ATF-2-IR and Fra-2-IR in CC $(p<0.001)$ (Table 1).

\section{Discussion}

It is suggested that DF which is a dense ectomesenchymal tissue surrounding the crown part of impacted teeth may be the cause of dentrigerous (follicular) cysts because of containing epithelial rests (Malassez epithelial rests) [23-25]. During the surgically extraction of impacted teeth, DF is recommended to be extracted under the opinion that it may cause cyst formation by undergoing pathological transformations [21].

In literature, there is not any sufficient information yet about the expression rate of AP-1 transcription factors under physiological conditions. Furthermore, no enough evidence regarding that there is a correlation between number of the cells which the proteins analyzed in this study are expressed in and general activity of the proteins in the tissue. In this study, given the known general characteristics of AP-1 transcription factors (like managing proliferation, differentiation and apoptosis in cells), the effects of AP-1 on cyst 
formation which is the most frequent pathological change of DF have been evaluated.

ATF-2 and Fra-2 which are the members of AP-1 transcription factors play important roles in growth, differentiation, apoptosis and cancer processes in cell [26-29]. In view of their cellular functions, it can be predicted that these proteins may be effective in cyst formation originating from epithelial cells of DF.

Since DF is disappeared when completed its function during healthy eruption of teeth, there is not any tissue we can compare as a control group for DF tissues in adults. Additionally, EC do not compose a regular epithelial tissue in DF. This situation raises difficulties in manual counts. Since EC is held responsible for cyst formation, in this study, CC was not separated according to its types, but all cells composing the tissue were counted.

According to the results of our study, when ATF-2IR is compared between EC and CC of DF, it is higher in $\mathrm{CC}$ than $\mathrm{EC}$, and therefore it is determined that ATF-2 protein expression may not be effective on epithelial-origin cyst formation in DF. When Fra-2-IR is compared between EC and $\mathrm{CC}$ of DF, Fra-2 protein expression is higher in $\mathrm{EC}$ than $\mathrm{CC}$, and therefore when the effect of Fra-2 expression on cellular processes is considered, it is determined that it may be more effective in cyst formation processes than ATF-2.

However, when ATF-2-IR and Fra-2-IR in each tissue is compared, despite a statistically significant difference not being found between the expressions of two proteins in EC weakens the hypothesis that Fra-2 may be effective on cyst formation, it should not be ignored that these proteins may show different effects at different rates. When considered that each one of AP-1 member proteins does not play an equal role in all of the cellular processes and each one is not expressed at similar rates for their effects on these processes [30], the possibility that Fra-2 protein which is expressed more in EC than CC may have a pathological effect in epithelial cells and may be responsible for cyst formation increases.

In Fra-2 -IR-positive cells in EC of DF, a more intensive nuclear staining was observed than ATF-2IR-positive cells. This intensive nuclear staining suggests that Fra-2 protein may has different effects on cellular and pathological processes in EC in comparison with ATF-2 protein. However, information relating to the pathobiological relevance of intracellular distribution of AP-1 proteins is insufficient and confused in the literature [31-33].

Observation of higher ATF-2-IR in CC of DF and the difference with Fra-2-IR being statistically significant $(p<0.001)$ has showed that ATF-2 may be more effective only in possible pathological processes originating from connective tissue of DF. Accordingly, while researching the influencing factors in the devel- opment of cyst or other pathologies originating from DF, the role of possible inductive effect of ATF-2-IR in connective tissue or inhibition of ATF-2 expression in prevention of the pathology should be researched.

Acknowledgement. The author wishes to thank to Dr. Dt. Humeyra Kocaelli from Istanbul University, Faculty of Dentistry, Department of Oral Surgery and Medicine due to her assistance in providing the tissues required for this study. This study was supported by Research Fund of the Istanbul University. (Project Number: T-101/15122006).

\section{References}

[ 1] Glauser DA, Brun T, Gauthier BR, Schlegel W. Transcriptional response of pancreatic beta cells to metabolic stimulation: large scale identification of immediate-early and secondary response genes. BMC Mol Biol. 2007;8:54.

[2] Pennypacker KR. AP-1 transcription factor complexes in CNS disorders and development. J Fla Med Assoc. 1995;82:551-554.

[3] Foletta VC. Transcription factor AP-1, and the role of Fra-2. Immunol Cell Biol. 1996;74:121-133.

[ 4] Andreucci JJ, Grant D, Cox DM, et al. Composition and function of AP-1 transcription complexes during muscle cell differentiation. J Biol Chem. 2002;277:16426-16432.

[ 5] Handel ML, Girgis L. Transcription factors. Best Pract Res Clin Rheumatol. 2001;15:657-675.

[6] Hess J, Angel P, Schorpp-Kistner M. AP-1 subunits: quarrel and harmony among siblings. $J$ Cell Sci. 2004;117:5965-5973.

[ 7] Karin M, Liu Z, Zandi E. AP-1 function and regulation. Curr Opin Cell Biol. 1997;9:240-246.

[ 8] Milde-Langosch K, Röder H, Andritzky B, et al. The role of the AP-1 transcription factors c-Fos, FosB, Fra-1 and Fra-2 in the invasion process of mammary carcinomas. Breast Cancer Res Treat. 2004;86:139-152.

[ 9] Yi JH, Park SW, Kapadia R, Vemuganti R. Role of transcription factors in mediating post-ischemic cerebral inflammation and brain damage. Neurochem Int. 2007;50:1014-1027.

[10] van Dam H, Castellazzi M. Distinct roles of Jun : Fos and Jun : ATF dimers in oncogenesis. Oncogene. 2001;20:2453-2464.

[11] Vlahopoulos SA, Logotheti S, Mikas D, Giarika A, Gorgoulis $\mathrm{V}$, Zoumpourlis V. The role of ATF-2 in oncogenesis. Bioessays. 2008;30:314-327.

[12] Milde-Langosch K, Janke S, Wagner I, et al. Role of Fra-2 in breast cancer: influence on tumor cell invasion and motility. Breast Cancer Res Treat. 2008;107:337-347.

[13] Wise GE, Huang H, Que BG. Gene expression of potential tooth eruption molecules in the dental follicle of the mouse. Eur J Oral Sci. 1999;107:482-486.

[14] Wise GE, Marks SC Jr, Zhao L. Effect of CSF-1 on in vivo expression of c-fos in the dental follicle during tooth eruption. Eur J Oral Sci. 1998;106:397-400.

[15] Wise GE, Zhao L, Lin F. Effects of epidermal growth factor (EGF) and colony-stimulating factor-1 (CSF-1) on expression of c-fos in rat mandibular molars: implications for tooth eruption. Cell Tissue Res. 1996;284:1-7.

[16] Kémoun P, Laurencin-Dalicieux S, Rue J, et al. Human dental follicle cells acquire cementoblast features under stimulation by BMP-2/-7 and enamel matrix derivatives (EMD) in vitro. Cell Tissue Res. 2007;329:283-294.

[17] Handa K, Saito M, Yamauchi M, et al. Cementum matrix formation in vivo by cultured dental follicle cells. Bone. 2002;31:606-611.

[18] Morsczeck C, Götz W, Schierholz J, et al. Isolation of precursor cells (PCs) from human dental follicle of wisdom teeth. Matrix Biol. 2005;24:155-165. 
[19] Conklin WW, Stafne EC. A study of odontogenic epithelium in the dental follicle. JADA. 1949;39:143-148.

[20] Edamatsu M, Kumamoto H, Ooya K, Echigo S. Apoptosisrelated factors in the epithelial components of dental follicles and dentigerous cysts associated with impacted third molars of the mandible. Oral Surg Oral Med Oral Pathol Oral Radiol Endod. 2005;99:17-23.

[21] Rakprasitkul S. Pathologic changes in the pericoronal tissues of unerupted third molars. Quintessence Int. 2001;32:633-638.

[22] Chindia ML. Pathogenesis of odontogenic cysts: an update. East Afr Med J. 1991;68:276-282.

[23] Benn A, Altini M. Dentigerous cysts of inflammatory origin. A clinicopathologic study. Oral Surg Oral Med Oral Pathol Oral Radiol Endod. 1996;81:203-209.

[24] Godoy GP, da Silveira EJ, Lins RD, de Souza LB, de Almeida Freitas R, Queiroz LM. Immunohistochemical profile of integrins in enlarged dental follicles and dentigerous cysts. Oral Surg Oral Med Oral Pathol Oral Radiol Endod. 2007;104:e29-34.

[25] Zeichner-David M, Oishi K, Su Z, et al. Role of Hertwig's epithelial root sheath cells in tooth root development. Dev Dyn. 2003;228:651-663.

[26] Keklikoglu N. c-Jun, Fra-2, and ATF-2 Immunoreactivity in the Jejunal Tissues of the Healthy Rat. Dig Dis Sci. 2008;53:2680-2686.

[27] Mason JM, Schmitz MA, Müller KM, Arndt KM. Semirational design of Jun-Fos coiled coils with increased affinity:
Universal implications for leucine zipper prediction and design. Proc Natl Acad Sci U S A. 2006;103:8989-8994.

[28] Nishikawa S. Localization of transcription factor AP-1 family proteins in ameloblast nuclei of the rat incisor. $J$ Histochem Cytochem. 2000;48:1511-1520.

[29] Tulchinsky E. Fos family members: regulation, structure and role in oncogenic transformation. Histol Histopathol. 2000;15:921-928.

[30] Keklikoglu N. The localization of Fos B, a member of transcription factor AP-1 family, in rat odontoblasts and pulpal undifferentiated ectomesenchymal cells. Folia Histochem Cytobiol. 2004;42:191-193.

[31] Zhang W, Hart J, McLeod HL, Wang HL. Differential expression of the AP-1 transcription factor family members in human colorectal epithelial and neuroendocrine neoplasms. Am J Clin Pathol. 2005;124:11-19.

[32] Malnou CE, Salem T, Brockly F, Wodrich H, Piechaczyk M, Jariel-Encontre I. Heterodimerization with Jun family members regulates c-Fos nucleocytoplasmic traffic. J Biol Chem. 2007;282:31046-31059.

[33] Berger AJ, Kluger HM, Li N, et al. Subcellular localization of activating transcription factor 2 in melanoma specimens predicts patient survival. Cancer Res. 2003;63:8103-8107.

Submitted: 16 October, 2010 Accepted after reveiws: 31 January, 2010 